\title{
Multi-Beam Multi-Wavelength Semiconductor Lasers
}

\section{Citation}

Yu, Nanfang, Mikhail A. Kats, Christian Pflügl, Markus Geiser, Qi Jie Wang, Mikhail A. Belkin, Federico Capasso, et al. 2009. Multi-beam multi-wavelength semiconductor lasers. In Lasers, Optics, and Optoelectronics. Special Issue. Applied Physics Letters 95(16): 161108.

\section{Published Version}

doi:10.1063/1.3253713

\section{Permanent link}

http://nrs.harvard.edu/urn-3:HUL.InstRepos:5349452

\section{Terms of Use}

This article was downloaded from Harvard University's DASH repository, and is made available under the terms and conditions applicable to Open Access Policy Articles, as set forth at http:// nrs.harvard.edu/urn-3:HUL.InstRepos:dash.current.terms-of-use\#OAP

\section{Share Your Story}

The Harvard community has made this article openly available.

Please share how this access benefits you. Submit a story.

Accessibility 


\title{
Multi-beam multi-wavelength semiconductor lasers
}

\author{
Nanfang Yu, ${ }^{1, a)}$ Mikhail A. Kats, ${ }^{1}$ Christian Pflügl, ${ }^{1}$ Markus Geiser, ${ }^{1}$ Qi Jie Wang, ${ }^{1, b)}$ \\ Mikhail A. Belkin, ${ }^{1, \mathrm{c})}$ Federico Capasso, ${ }^{1, \mathrm{~d})}$ Milan Fischer, ${ }^{2}$ Andreas Wittmann, ${ }^{2}$ \\ Jérôme Faist, ${ }_{3}{ }^{3}$ Tadataka Edamura, ${ }^{3}$ Shinichi Furuta, ${ }^{3}$ Masamichi Yamanishi, ${ }^{3}$ and \\ Hirofumi Kan ${ }^{3}$ \\ ${ }^{1}$ School of Engineering and Applied Sciences, Harvard University, Cambridge, Massachusetts 02138, USA \\ ${ }^{2}$ Institute of Quantum Electronics, ETH Zürich, CH-8093 Zürich, Switzerland \\ ${ }^{3}$ Central Research Laboratory, Hamamatsu Photonics K.K., Hamamatsu 434-8601, Japan
}

(Received 15 July 2009; accepted 2 October 2009; published online 23 October 2009; publisher error corrected 28 October 2009)

\begin{abstract}
Multibeam emission and spatial wavelength demultiplexing in semiconductor lasers by patterning their facets with plasmonic structures is reported. Specifically, a single-wavelength laser was made to emit beams in two directions by defining on its facet two metallic gratings with different periods. The output of a dual-color laser was spatially separated according to wavelength by using a single metallic grating. The designs can be integrated with a broad range of active or passive optical components for applications such as interferometry and demultiplexing. (C) 2009 American Institute of Physics. [doi:10.1063/1.3253713]
\end{abstract}

Beam engineering of semiconductor light sources by patterning plasmonic structures directly on their emission facet has been actively pursued in recent years. ${ }^{1-10}$ This approach allows for the creation of light sources with enhanced performance and/or new functionalities. These sources can then be used for a variety of applications without resorting to external optical components for beam shaping. For example, plasmonic structures have been used to enhance directionality and increase the emitted optical power of light-emitting diodes. ${ }^{4,5}$ The problem of polarization instability in verticalcavity surface-emitting lasers (VCSELs) has been addressed by patterning anisotropic plasmonic patterns on the device facet. ${ }^{6,7}$ Plasmonic collimators have been shown to reduce beam divergence of edge-emitting quantum cascade lasers (QCLs) by orders of magnitude while preserving their power output. ${ }^{8,9}$ Plasmonics have been demonstrated as a promising and technologically simple approach for achieving polarization control in semiconductor lasers. ${ }^{10}$

In this letter, we demonstrate multibeam emission and spatial wavelength demultiplexing using plasmonic structures. We report simulations and experimental results for two different lasers. In the first, a single-wavelength QCL produces two small-divergence beams with approximately equal peak intensity. In the second, a QCL emits two wavelengths in different directions.

Previously, a suitably designed aperture-grating plasmonic structure on a QCL facet was shown to collimate the emerging beam. ${ }^{8,9}$ The subwavelength aperture defined on the laser active region couples a substantial part of the laser output into propagating surface plasmons (SPs). If the period of the plasmonic grating is approximately equal to the SP wavelength, the waves scattered from the grating grooves

\footnotetext{
${ }^{a)}$ Electronic mail: nyu@fas.harvard.edu.

${ }^{b}$ Present address: School of Electrical and Electronic Engineering \& School of Physical and Mathematical Sciences, Nanyang Technological University, Nanyang Ave, 639798 Singapore.

${ }^{c}$ Present address: Department of Electrical and Computer Engineering, The University of Texas at Austin, Austin, Texas 78758, USA.

${ }^{\mathrm{d})}$ Electronic mail: capasso@ seas.harvard.edu.
}

and the aperture interfere constructively in the far field, yielding a collimated beam normal to the laser facet. ${ }^{8}$ If instead the grating period differs from the SP wavelength, waves from neighboring grooves will have a constant phase difference (different from $2 \pi$ ) and constructive interference will occur in a direction away from the normal of the facet. ${ }^{11-14}$

We utilized this concept to split the emission of a single wavelength QCL into two collimated beams in different directions. Two successive plasmonic gratings with different periods and lengths were defined on the device facet. By tuning the grating period, the emission direction of the beam originating from each individual grating can be controlled; by tuning the grating length (i.e., the number of grooves per grating), we can independently control the intensity of the emitted beams. Note that since SPs are able to propagate for significant distances comparable to approximately 1000 wavelengths in the mid-infrared region; ${ }^{15}$ several gratings can therefore be patterned on the laser facet to produce multiple laser beams with controlled intensity and direction.

To design the structure, numerous rapid two-dimensional (2D) simulations using a finite element electromagnetic solver (COMSOL) were performed. After a promising design was found, it was verified by a full three-dimensional simulation using a finite-difference time-domain solver (Lumerical FDTD) running on a computational cluster.

Figure 1 shows experimental and simulation results for a single-wavelength laser emitting two beams. The grating closer to the aperture is designed to produce a beam propagating normal to the facet (i.e., grating period approximately equal to the SP wavelength). The grating farther away from the aperture has a smaller grating period [Fig. 1(a)]. It provides a reciprocal lattice vector larger than the wavevector of the SPs, and therefore light scattered from the grating grooves is deflected away from the normal toward the top electrical contact of the device. The deflection angle is designed to be approximately $20^{\circ}$ from the surface normal, which is confirmed by simulations and experiments; see 2D far-field patterns in Fig. 1(b) and their line scans in Fig. 1(c). The 2D simulation, right half of Fig. 1(b), is in good agree- 

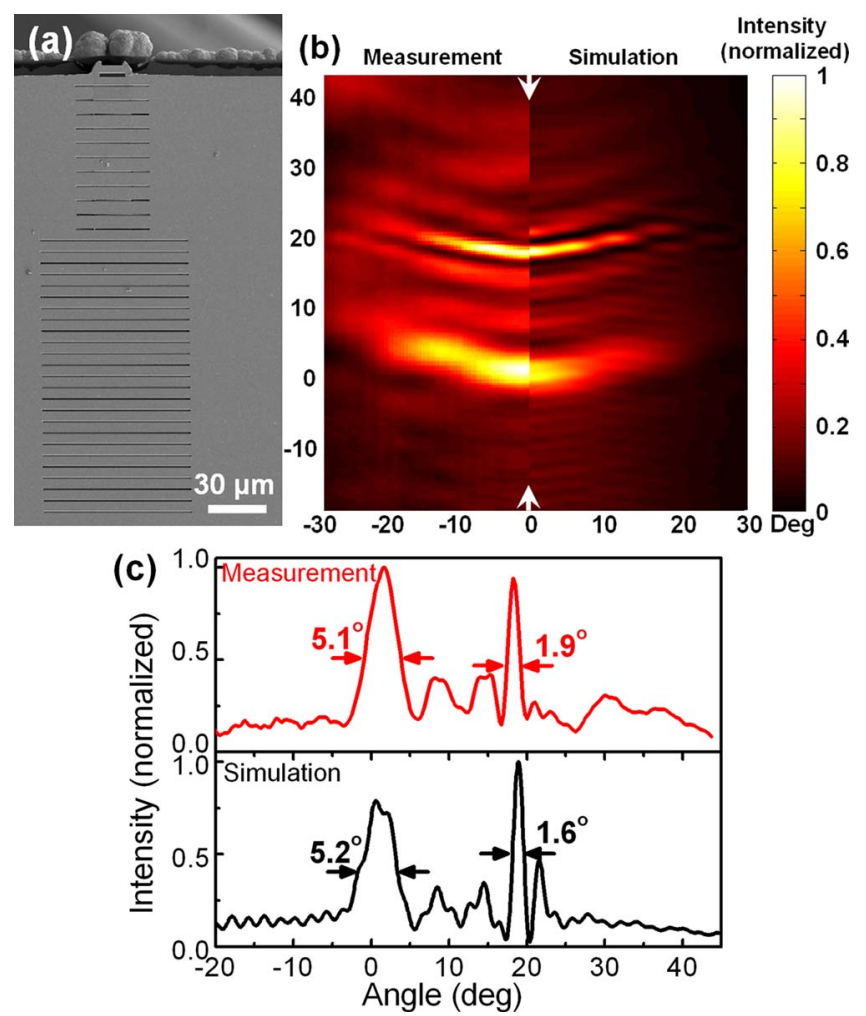

FIG. 1. (Color online) (a) Electron micrograph of a $\lambda=8.06 \mu \mathrm{m}$ QCL patterned with two gratings. The grating closer to the aperture contains 11 grooves and has a $7.8 \mu \mathrm{m}$ period; the other grating contains 25 grooves and has a $6 \mu \mathrm{m}$ period. The width and depth of the grooves are 0.6 and $1 \mu \mathrm{m}$, respectively. The thickness of gold is about $200 \mathrm{~nm}$. (b) Measured (left half) and simulated (right half) far-field emission patterns of the device. (The far-field patterns are symmetric so only half of them are shown.) (c) Vertical line scans of the measured (upper panel) and the simulated (lower panel) far-field pattern along the arrows in (b).

ment with the measured far-field, the left half of Fig. 1(b), including its curved features of the far-field patterns, which are due to edge effects originating from the finite lateral size of the aperture and gratings. ${ }^{8}$

Since the intensity of SPs decreases as it propagates through the grating grooves due to both scattering from the grooves and losses within the metal, the second grating should contain more grooves than the first one to allow the two emitted beams to have the same peak intensity. Indeed, by including 11 and 25 grooves in the two gratings, respectively, we see from the line scans in Fig. 1(c) that the two lobes are approximately equal in peak intensity. The measured peak intensity of the narrower beam [upper panel of Fig. 1(c)] is slightly smaller than that predicted by simulations [lower panel of Fig. 1(c)]. This is most likely due to additional attenuation of SPs originating from scattering by inevitable surface roughness introduced in the fabrication process. This effect attenuates SPs before reaching the second grating. The two beams have different divergence, see Fig. 1(c), because the divergence angle is roughly inversely proportional to the number of the grooves in the grating. ${ }^{8}$

The power output versus driving current characteristics (LI curves) of the device are presented in Fig. 2. Its peak output power is $\sim 60 \%$ of that of the original device. The power output was measured using a calibrated thermopile detector that collects laser emission via a metallic tube right in front of the device facet.

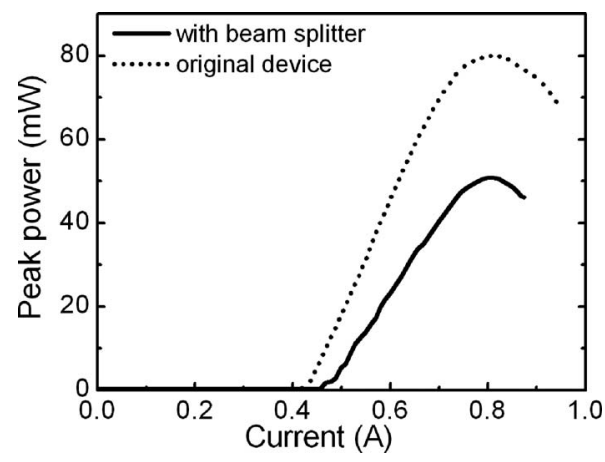

FIG. 2. LI characteristics taken before (dotted curve) and after (solid curve) patterning the plasmonic beam splitter for the device shown in Fig. 1.

For the lasers emitting spatially demultiplexed wavelengths, we used mid-infrared QCLs with two stacks of active regions: one based on a two-phonon design emitting at $\sim 10.5 \mu \mathrm{m}$ and the other based on a bound-to-continuum (BTC) design emitting at $\sim 9.3 \mu \mathrm{m} \cdot{ }^{16,17}$ It is essential to devise a plasmonic grating structure that is efficient in scattering light at both wavelengths. Optimal grating geometry for one wavelength will not necessarily give good collimation for another wavelength because collimation is especially sensitive to the depth and width of the grating grooves. ${ }^{18} \mathrm{We}$ chose a design that is the trade-off between the optimal structures for the two wavelengths: the groove depth (width) is 1.3(0.7) $\mu \mathrm{m}$, while the optimal groove depth (width) for $\lambda$ $=9.3$ and $10.5 \mu \mathrm{m}$ is $1.1(0.7) \mu \mathrm{m}$ and 1.6(0.7) $\mu \mathrm{m}$, respectively. We used a single grating with a period of $8.5 \mu \mathrm{m}$ which deflects the $\lambda=9.3 \mu \mathrm{m}$ component away from the facet normal toward the top contact by $\sim 3^{\circ}$ and the $\lambda$ $=10.5 \mu \mathrm{m}$ component by $\sim 12^{\circ}$.

Simulations and experimental results for a dualwavelength device are presented in Fig. 3. The grating contains 30 grooves; see Fig. 3(a). A $9.5 \mu \mathrm{m}$ short-pass filter was placed in front of the midinfrared detector to allow mapping of the far-field pattern of the $\lambda=9.3 \mu \mathrm{m}$ component; the result is shown in the left half of Fig. 3(b). The emission pattern of the $\lambda=10.5 \mu \mathrm{m}$ component is shown in the left half of Fig. 3(c), which is obtained by subtracting the contribution of the $\lambda=9.3 \mu \mathrm{m}$ component from the far-field pattern measured using the detector without the filter. The measured direction and divergence angle of the two beams are in agreement with simulations; see Fig. 3(d) for the vertical line scans of Figs. 3(b) and 3(c). Our original unpatterned device lased on the fundamental $\mathrm{TM}_{00}$ mode for both wavelengths with only one lasing spot as confirmed by far-field measurements. After patterning the plasmonic beam demultiplexer, the two components still lase in the $\mathrm{TM}_{00}$ mode corresponding to the single lobes in Figs. 3(b) and 3(c).

The LI curves of the dual-wavelength device before and after defining the plasmonic demultiplexer are plotted in Fig. 4. For the original laser, the $\lambda=10.5 \mu \mathrm{m}$ component has a lower threshold current and an approximately five times larger slope efficiency than the $\lambda=9.3 \mu \mathrm{m}$ component. With the beam demultiplexer, the laser threshold of the $\lambda$ $=10.5 \mu \mathrm{m}$ component increases from about 1.5 to $1.75 \mathrm{~A}$ as a result of increased losses due to the scattering of laser light at the metallic aperture on the facet. However, the laser threshold of the $\lambda=9.3 \mu \mathrm{m}$ component does not change significantly (but is still higher than that of the other compo- 

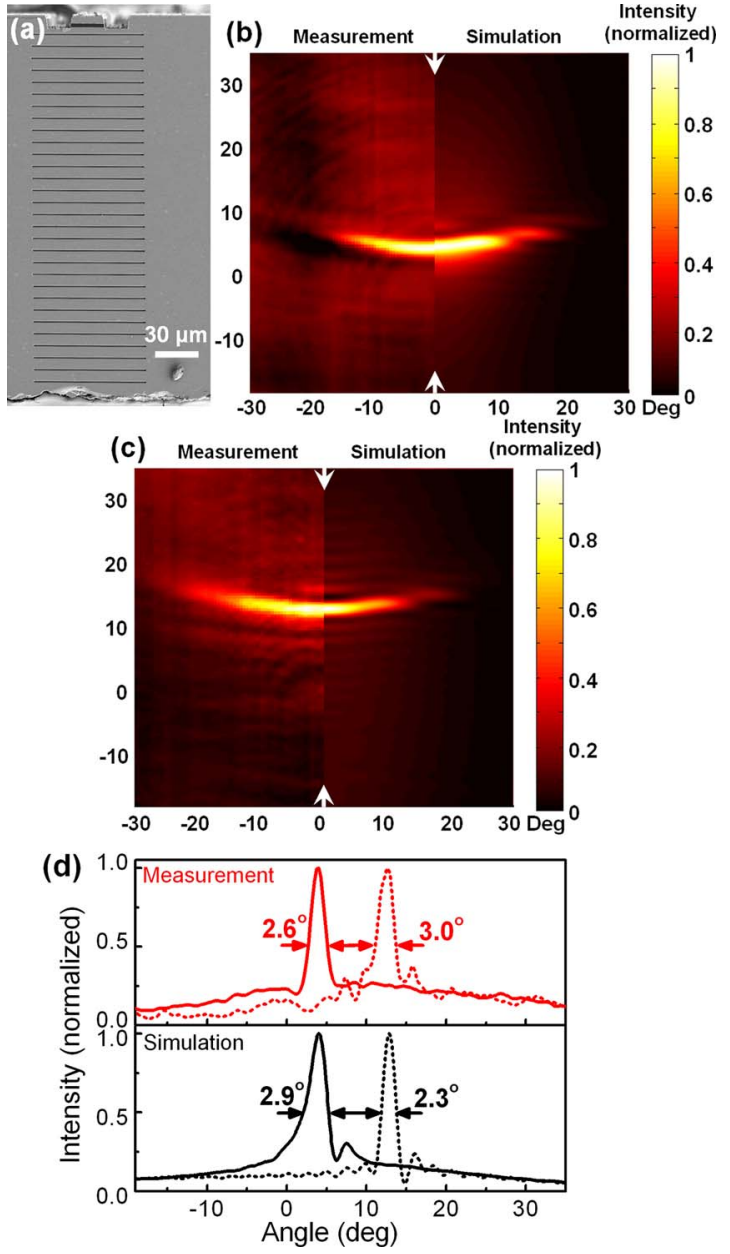

FIG. 3. (Color online) (a) Electron micrograph of a dual-wavelength QCL patterned with a demultiplexer. (b) Measured (left half) and simulated (right half) far-field emission patterns of the device at $\lambda=9.3 \mu \mathrm{m}$. and (c) Measured (left half) and simulated (right half) far-field emission pattern of the device at $\lambda=10.5 \mu \mathrm{m}$. (d) Vertical line scans of (b) and (c) along the arrows. Upper and lower panels are, respectively, experimental and simulated results. Solid curves and dotted curves are for the $\lambda=9.3 \mu \mathrm{m}$ and $\lambda$ $=10.5 \mu \mathrm{m}$ components, respectively.

nent). This is most likely due to two effects that are cancelling each other out. On one hand, scattering at the aperture increases the losses. On the other hand, the interplay of the two components sharing the same waveguide has to be considered. The $\lambda=10.5 \mu \mathrm{m}$ component starts lasing first and reduces the population inversion of the $\lambda=9.3 \mu \mathrm{m}$ BTC stack due to stimulated emission that reduces the gain at $\lambda$ $=9.3 \mu \mathrm{m} .{ }^{19}$ This effect is weaker in the device with the beam demultiplexer than in the original device due to decreasing optical power of the $\lambda=10.5 \mu \mathrm{m}$ component. The power throughput of the device after patterning the demultiplexer is $\sim 50 \%-60 \%$ of that of the original device for the two wavelengths.

The beam splitter for single-wavelength lasers discussed in this letter can be potentially useful for applications requiring a reference beam and a probe beam such as interferometry and holography. Our designs should be scalable and applicable to near-infrared lasers and passive optical components. For example, the spatial wavelength demultiplexing component for dual-wavelength lasers can function

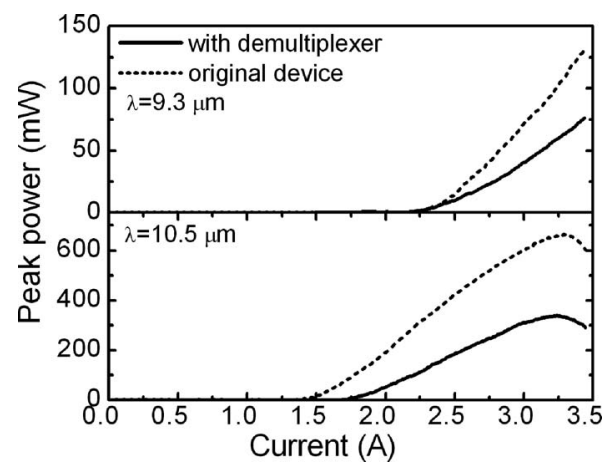

FIG. 4. LI characteristics of the dual-wavelength device measured before (dotted curves) and after patterning the demultiplexer (solid curves).

as a demultiplexer for optical fiber communications.

We acknowledge support from the Air Force Office of Scientific Research (AFOSR MURI on Plasmonics) and the Harvard Nanoscale Science and Engineering Center (NSEC). This work was performed in part at the Center for Nanoscale Systems (CNS) at Harvard University, a member of the National Nanotechnology Infrastructure Network (NNIN), which is supported by the National Science Foundation. This research benefited from the research computing resources at the Harvard School of Engineering and Applied Sciences.

${ }^{1}$ F. Capasso, N. Yu, E. Cubukcu, and E. Smythe, Opt. Photonics News, May issue, 22 (2009).

${ }^{2}$ E. Cubukcu, E. A. Kort, K. B. Crozier, and F. Capasso, Appl. Phys. Lett. 89, 093120 (2006).

${ }^{3}$ N. Yu, E. Cubukcu, L. Diehl, M. A. Belkin, K. B. Crozier, F. Capasso, D. Bour, S. Corzine, and G. Höfler, Appl. Phys. Lett. 91, 173113 (2007).

${ }^{4}$ K. Okamoto, I. Niki, A. Shvartser, Y. Narukawa, T. Mukai, and A. Scherer, Nature Mater. 3, 601 (2004).

${ }^{5}$ M. D. Harries and H. D. Summers, IEEE Photonics Technol. Lett. 18, 2197 (2006).

${ }^{6}$ J.-H. Ser, Y.-G. Ju, J.-H. Shin, and Y. H. Lee, Appl. Phys. Lett. 66, 2769 (1995).

${ }^{7}$ P. Babu Dayal and F. Koyama, Appl. Phys. Lett. 91, 111107 (2007).

${ }^{8}$ N. Yu, J. Fan, Q. J. Wang, C. Pflügl, L. Diehl, T. Edamura, M. Yamanishi, H. Kan, and F. Capasso, Nat. Photonics 2, 564 (2008).

${ }^{9}$ N. Yu, R. Blanchard, J. Fan, Q. J. Wang, C. Pflügl, L. Diehl, T. Edamura, M. Yamanishi, H. Kan, and F. Capasso, Opt. Express 16, 19447 (2008).

${ }^{10}$ N. Yu, Q. J. Wang, C. Pflügl, L. Diehl, F. Capasso, T. Edamura, S. Furuta, M. Yamanishi, and H. Kan, Appl. Phys. Lett. 94, 151101 (2009).

${ }^{11}$ H. J. Lezec, A. Degiron, E. Devaux, R. A. Linke, L. Martín-Moreno, F. J. García-Vidal, and T. W. Ebbesen, Science 297, 820 (2002).

${ }^{12}$ J. Bravo-Abad, F. J. Garćia-Vidal, and L. Martín-Moreno, Photonics Nanostruct. Fundam. Appl. 1, 55 (2003).

${ }^{13}$ L. -B. Yu, D. -Z. Lin, Y. -C. Chen, Y. -C. Chang, K. -T. Huang, J. -W. Liaw, J. -T. Yeh, J. -M. Liu, C. -S. Yeh, and C. -K. Lee, Phys. Rev. B 71, 041405(R) (2005)

${ }^{14}$ S. Kim, H. Kim, Y. Lim, and B. Lee, Appl. Phys. Lett. 90, 051113 (2007)

${ }^{15}$ N. Yu, R. Blanchard, J. Fan, Q. J. Wang, C. Pflügl, L. Diehl, T. Edamura, S. Furuta, M. Yamanishi, H. Kan, and F. Capasso, "Plasmonics for laser beam shaping," IEEE Trans. Nanotechnol. (to be published).

${ }^{16}$ M. A. Belkin, F. Capasso, F. Xie, A. Belyanin, M. Fischer, A. Wittmann, and J. Faist, Appl. Phys. Lett. 92, 201101 (2008).

${ }^{17}$ C. Pflügl, M. A. Belkin, Q. J. Wang, M. Geiser, A. Belyanin, M. Fischer, A. Wittmann, J. Faist, and F. Capasso, Appl. Phys. Lett. 93, 161110 (2008)

${ }^{18}$ L. Martín-Moreno, F. J. García-Vidal, H. J. Lezec, A. Degiron, and T. W. Ebbesen, Phys. Rev. Lett. 90, 167401 (2003).

${ }^{19}$ M. Geiser, C. Pflügl, Q. J. Wang, F. Capasso, A. Belyanin, T. Edamura, and H. Kan (unpublished). 